\title{
La variedad y la calidad de las exportaciones españolas*
}

\author{
Xavier Cuadras Morató \\ Departamento de Economía y Empresa \\ y Escola Superior de Comerç Internacional \\ Universitat Pompeu Fabra \\ Jordi Puig Gabau \\ Escola Superior de Comerç Internacional \\ Universitat Pompeu Fabra
}

\section{Resumen}

Dentro del contexto europeo la economía española es una de las que tiene un porcentaje de exportaciones en relación al PIB más bajo. El objetivo de este trabajo es averiguar si esto se debe a una menor variedad de productos exportados (margen extensivo), un menor valor de los productos efectivamente exportados (margen intensivo) o al hecho de que los productos exportados son de menor calidad y precio (margen cualitativo). Los resultados de nuestro análisis revelan que los tres márgenes contribuyen de forma relevante a la hora de explicar este fenómeno. La utilización de los datos desagregados de las exportaciones españolas nos permite también analizar la posición exportadora de las principales economías regionales.

Palabras clave: exportaciones, margen intensivo, margen extensivo, margen cualitativo.

Clasificación JEL: F14.

\begin{abstract}
Within the European context the Spanish economy has a comparatively low value for exports as a percentage of GDP. The objective of the current paper is to find out whether this is due to a smaller variety of exports (extensive margin), a smaller value of products actually exported (intensive margin) or the fact that exports are of a lower quality and price (qualitative margin). The outcome of our analysis reveals that the three margins make a positive contribution in order to explain this phenomenon. The use of disaggregated data of the Spanish exports allows us to analyze the exporting position of the main regional economies.
\end{abstract}

Keywords:

JEL classification: F14.

${ }^{*}$ Los autores del trabajo desean expresar su agradecimiento al Observatori dels Mercats Exteriors del COPCA (Generalitat de Catalunya) por su apoyo financiero para la realización de este trabajo, al director de la revista, Sergi Jiménez-Martín y un evaluador anónimo por sus valiosos comentarios y a Andrés Álvarez y Carles Galofré por su labor como ayudantes de investigación. Xavier Cuadras Morató también agradece el apoyo financiero del Ministerio de Educación y Ciencia (proyectos SEJ200403149 y SEJ2007-64340). 


\section{Introducción}

Si se compara el valor de las exportaciones de distintos países, las causas de las diferencias que pueden observarse se acaban reduciendo a dos tipologías obvias. Por una parte, puede que los mayores exportadores vendan mayores valores de cada producto (lo que se denomina el margen intensivo). Alternativamente, puede que esos países exporten un mayor número de productos (el margen extensivo) ${ }^{1}$. Lógicamente lo más frecuente es que se produzca una combinación de ambos márgenes. A su vez, el margen intensivo puede descomponerse en dos elementos, puesto que el mayor valor de las exportaciones puede deberse al hecho de que se exportan mayores cantidades físicas de cada bien o al hecho de que el precio de los bienes exportados es más alto. Dado que en el contexto del comercio internacional precios más altos normalmente corresponden a bienes de mayor calidad, se ha dado en llamar a esta última circunstancia el margen cualitativo de las exportaciones.

A pesar del importante crecimiento de las exportaciones experimentado en los últimos años ${ }^{2}$, la economía española es todavía una de las economías europeas que tiene un porcentaje de exportaciones en relación al PIB más bajo (un 15\% comparado con el $27 \%$ que corresponde al total de todos los países de la UE25 en el año $2005)^{3}$. Siendo éste un dato sobradamente conocido, es interesante identificar qué márgenes de las exportaciones son sus principales responsables, en el contexto de un estudio comparado con el resto de los países europeos. Además, la existencia de datos sobre exportaciones regionales en España permite profundizar en posibles explicaciones de este fenómeno a un nivel más desagregado. Así, el principal objetivo de este trabajo es presentar y analizar los datos correspondientes a la descomposición en los márgenes referidos de las exportaciones de los países europeos y de las principales regiones españolas exportadoras (Cataluña, Madrid, País Vasco, Valencia, Andalucía y Galicia $)^{4}$ para el año 2005. Los distintos márgenes calculados se analizan en relación a una serie de características relevantes de las economías estudiadas, de acuerdo con las previsiones de algunos de los modelos de comercio internacional más comunes en la literatura. El trabajo nos permite posicionar por primera vez los már-

${ }^{1}$ No debe confundirse el sentido del término margen extensivo que se utiliza en este trabajo y todas las referencias que se incluyen con los sentidos que también se han utilizado en otros trabajos en contextos similares. El primero de estos sentidos sería el referido al número de mercados de destino de las exportaciones. En este caso el crecimiento del margen extensivo se referiría al desarrollo de nuevos mercados que antes no habían sido explotados (véanse referencias muy recientes en BESEDES y PRUSA, 2006 y FELBERMAYR y KOHLER, 2006). El segundo se refiere al número de empresas exportadoras (véanse referencias muy recientes en CHANEY, 2006, y HELPMAN, MELITZ y RUBINSTEIN, 2007).

2 Durante el período 1993-2005 las exportaciones españolas crecieron a una tasa anual acumulativa del $10,5 \%$, mientras que crecimiento en el mismo período para el total de los países de la UE25 fue del 8,7 \% (datos procedentes de DataComex, Ministerio de Industria, Turismo y Comercio).

3 Datos extraídos de Eurostat.

${ }^{4}$ Las seis regiones referidas exportan más del $76 \%$ del total correspondiente a la economía española. La mayor exportadora de las regiones no consideradas (Castilla-León) en el análisis exporta menos del $6 \%$ del total español. 
genes exportadores de las principales economías regionales españolas en relación a la situación que se produce en los países del entorno más inmediato.

Recientemente se han desarrollado líneas de investigación en temas de comercio internacional que vienen a sugerir que los márgenes extensivo y cualitativo son mucho más importantes en el análisis de los flujos comerciales de lo que tradicionalmente se había pensado. Contribuciones capitales en este sentido son las de Hummels y Klenow (2005), Kehoe y Ruhl (2003) y Schott (2004). Partiendo de la observación de que las economías más grandes son también las economías más exportadoras, Hummels y Klenow (2005) descomponen las exportaciones de un número importante de países entre las contribuciones correspondientes a los márgenes extensivo, intensivo y cualitativo con datos procedentes de 126 países para el año 1995 y relacionan cada uno de estos márgenes con el tamaño de las economías (medido a través del PIB) y sus componentes (número de trabajadores empleados y PIB por empleado). El interés de este ejercicio es muy relevante por cuanto que, aunque los principales modelos de comercio internacional coinciden en la predicción de que mayores economías generan mayores exportaciones, la contribución de los distintos márgenes de las exportaciones es muy distinta según las diferentes teorías analizadas. Así, mientras las teorías basadas en el modelo de diferenciación nacional de Armington (1969) ponen el acento en el margen intensivo, los modelos de competencia monopolística (Krugman, 1981, por ejemplo) enfatizan el margen extensivo y las teorías basadas en la diferenciación vertical (como Grossman y Helpman, 1991) subrayan la importancia del margen cualitativo. Los principales resultados de Hummels y Klenow (2005) se pueden resumir de la manera siguiente. En primer lugar, un 60\% de las mayores exportaciones de las economías más grandes se deben a la contribución del margen extensivo, siendo el $40 \%$ restante la contribución del margen intensivo. Asimismo, la contribución del margen intensivo está dominada por las mayores cantidades de cada bien por encima de los precios unitarios más altos, aunque los países más ricos (PIB por empleado más alto) sí exportan productos de mayor calidad y precio.

Por su parte, Kehoe y Ruhl (2003) estudian las relaciones comerciales de 18 países (los tres países firmantes del NAFTA y los quince países de la UE antes de las ampliaciones) durante algunos procesos de liberalización comercial importantes como son la creación del NAFTA en 1994 o la puesta en marcha del mercado único europeo en $1993^{5}$. Sus conclusiones principales van en la línea de demostrar que la contribución del margen extensivo al crecimiento de las exportaciones después de un proceso de liberalización comercial es muy significativa, un hecho que, por otra parte, es difícil de explicar en la mayoría de los modelos teóricos más utilizados en el análisis del comercio internacional, al menos hasta la reciente contribución de Melitz (2003) ${ }^{6}$.

5 Para el caso del NAFTA, se puede ver también HILLBERRY y MCDANIEL (2002).

${ }^{6}$ MELITZ (2003) es el punto de partida teórico de una serie de modelos recientes que sí vinculan los procesos de liberalización comercial con el aumento de las exportaciones de nuevos productos. Entre otros cabe destacar los trabajos de AMURGO-PACHECO (2006), BALDWIN y DININO (2006) y AMURGO-PACHECO y PIEROLA (2007). 
Finalmente, Schott (2004) es una de las principales referencias sobre la importancia del crecimiento del margen cualitativo en el comercio internacional. Este trabajo documenta como los Estados Unidos cada vez más importan los mismos productos procedentes tanto de países desarrollados como de países en vías de desarrollo. No obstante, los precios unitarios de estos productos (factor relacionado con la calidad) varían sustancialmente según los países de procedencia, poniéndose en evidencia la existencia de una división «vertical» intrasectorial del trabajo a nivel internacional, en la que los países ricos están especializados en bienes de precio alto y calidad elevada y los países pobres exportan variedades de los mismos bienes, pero de menor calidad y precio.

El número de trabajos que documentan empíricamente la importancia de los márgenes extensivo y cualitativo es creciente y, entre otros, cabe destacar los siguientes: Evenett y Venables (2002) estudian el crecimiento de las exportaciones de un grupo de veintiséis países en desarrollo y de renta media para el período 1970-97; Amurgo Pacheco (2006) explora el caso del comercio entre los países europeos y mediterráneos; Debaere y Mostashari (2005) estudian la influencia directa de las reducciones arancelarias en el desarrollo del margen extensivo del comercio; Kang (2004) demuestra la importancia del margen extensivo para los casos de las exportaciones de Corea y Taiwan; Flam y Nordström (2006) examinan el impacto de la introducción del euro y documentan la mayor importancia del margen extensivo sobre el intensivo en el crecimiento del comercio que se deriva de este fenómeno; Funke y Ruhwedel (2003) hacen los mismo para las economías en transición de los países de Europa Oriental y Central; Feenstra y Kee (2007) se centran en los casos de las exportaciones de Méjico y China a los Estados Unidos; Fabrizio, Igan y Mody (2007) estudian el papel de la mejora de la calidad de las exportaciones en el crecimiento de la cuota de mercado en el comercio mundial de los ocho países de la Europa del Este que se incorporaron a la Unión Europea en el año 2004; y, por último, Faruq (2006) se pregunta por qué los países exportan productos de calidades diferentes y examina el papel que tiene en la respuesta la tecnología y la inversión directa extranjera.

En este trabajo se utiliza la metodología propuesta por Hummels y Klenow (2005) para determinar los márgenes intensivo, extensivo y cualitativo de las exportaciones y se aplica a los datos correspondientes al año 2005 de los 25 países que formaban la Unión Europea ese año, además de las seis regiones españolas con mayor capacidad exportadora. El análisis de estos datos permite evaluar no sólo la situación relativa de España como país exportador en relación al resto de los países de la UE, sino también las posiciones relativas del grupo más significativo de economías regionales exportadoras. Aunque España también estaba incluida en el grupo de 126 países estudiado por Hummels y Klenow (2005), es interesante comprobar si tras diez años de importante crecimiento de las exportaciones españolas las conclusiones del susodicho trabajo con respecto a la economía española han variado de forma sustancial. 
Los principales resultados que se derivan del trabajo se pueden resumir de la manera siguiente. En primer lugar, el margen extensivo es responsable de una tercera parte de las mayores exportaciones de las economías europeas más grandes, correspondiendo las dos terceras partes restantes al margen intensivo. Con respecto a la descomposición del margen intensivo en precios y cantidades, se detecta que los países con mayor PIB en términos absolutos exportan mayores cantidades, a precios que no son significativamente más elevados. En cambio, países más ricos, es decir, con mayores valores de PIB por ocupado, no exportan cantidades significativamente mayores, pero lo hacen a precios de las exportaciones que sí que son significativamente más altos. En relación a los resultados correspondientes a España y las regiones españolas consideradas, es evidente que la economía española tiene un peso exportador bastante menor de lo que cabría esperar dado su tamaño. Además, todas las economías regionales presentan índices exportadores que están por debajo de lo que les correspondería según su dimensión. En términos generales, los dos márgenes, intensivo y extensivo, se reparten la responsabilidad del escaso peso exportador español. La variabilidad regional respecto de este resultado es más bien escasa. Los márgenes intensivo y extensivo para las seis comunidades autónomas analizadas son relativamente reducidos, con las excepciones del margen extensivo para el caso de Cataluña y el margen intensivo para Galicia y, en menor medida, el País Vasco. En relación al margen cualitativo, destaca el relativamente bajo nivel de precios de las exportaciones españolas (agregadas y por regiones) cuando se comparan con economías similares en términos de PIB por ocupado, siendo las únicas excepciones a esta regla las economías de Madrid y Andalucía.

El resto del artículo se estructura de la forma siguiente. La sección 2 detalla la metodología seguida y la base de datos utilizada. La sección 3 describe y analiza los principales resultados de nuestra investigación y, finalmente, la sección 4 presenta las principales conclusiones del trabajo.

\section{Metodología y base de datos}

Hummels y Klenow (2005) evalúan la importancia de los márgenes extensivo, intensivo y cualitativo de las exportaciones de 126 países dirigidas hacia 59 mercados importadores en el año 1995. El presente trabajo pretende utilizar la metodología desarrollada en el artículo referido para evaluar la composición de las exportaciones en el año 2005 de los 25 países de la Unión Europea antes de la ampliación del año 2007 más las seis comunidades autónomas españolas mencionadas anteriormente hacia los 67 destinos cuantitativamente más importantes de estas exportaciones (representan el 97,27\% del total de les exportaciones de los países de la UE25 del año 2005) ${ }^{7}$.

7 Más detalles sobre la metodología empleada se pueden consultar en HUMMELS y KLENOW (2005) y también en HUMMELS y KLENOW (2002). 
El margen extensivo de las exportaciones del país $j(j=\{1, \ldots, 31\})$ hacia el mercado $m(m=\{1, \ldots, 67\})$ se define de la siguiente manera:

$$
M E_{j m}=\frac{\sum_{i \in I_{j m}} p_{k m i} x_{k m i}}{\sum_{i \in I} p_{k m i} x_{k m i}}
$$

donde $I_{j m}$ es el conjunto de categorías (productos) en los que el país $j$ tiene exportaciones positivas hacia el país $m, I$ es el conjunto de todas las categorías en las que la totalidad de los países de la UE25 tienen exportaciones hacia el país $m$ y $p_{k m i} x_{k m i}$ es el valor monetario de las exportaciones del producto $i$ de la economía de referencia $k$ (en nuestro caso $k$ es la UE25) hacia el mercado $m$. Podemos tomar como ejemplo las exportaciones francesas hacia Australia. El margen extensivo de las exportaciones del país $j$ (Francia) hacia el mercado $m$ (Australia) sería equivalente al porcentaje que representan del total de las exportaciones de los países de la UE25 al mercado $m$ (Australia) aquellas exportaciones que corresponden a los productos que el país $j$ (Francia) exporta hacia Australia.

De manera similar el margen intensivo vendría definido por

$$
M I_{j m}=\frac{\sum_{i \in I_{j m}} p_{j m i} x_{j m i}}{\sum_{i \in I_{j m}} p_{k m i} x_{k m i}}
$$

donde $p_{j m i} x_{j m i}$ es el valor monetario de las exportaciones del producto $i$ de la economía $j$ (en nuestro ejemplo, Francia) hacia el mercado $m$ (Australia). El margen intensivo equivaldría al porcentaje que representan las exportaciones francesas hacia Australia del total de las exportaciones de la UE25, sólo considerando aquellos productos exportados por Francia hacia el mercado australiano. Evidentemente el producto de los márgenes extensivo e intensivo definidos de esta forma corresponde a la cuota francesa de todas las exportaciones de la UE25 hacia Australia.

A continuación se procede a explicar la descomposición del margen intensivo en dos índices que corresponden a precios $\left(P_{j m}\right)$ y cantidades $\left(X_{j m}\right)$, de acuerdo con la expresión $M I_{j m}=P_{j m} X_{j m} \mathrm{y}$ donde el índice de precios viene definido por la media geométrica

$$
P_{j m}=\prod_{i \in I_{j m}}\left(\frac{p_{j m i}}{p_{k m i}}\right)^{w_{j m i}}
$$

donde el factor de ponderación $w_{j m i}$ viene dado por la expresión:

$$
w_{j m i}=\frac{\frac{s_{j m i}-s_{k m i}}{\ln s_{j m i}-\ln s_{k m i}}}{\sum_{i \in I_{j m}} \frac{s_{j m i}-s_{k m i}}{\ln s_{j m i}-\ln s_{k m i}}}
$$


es decir, la media logarítmica de $s_{j m i}$ y $s_{k m i}$, las cuales representan, respectivamente, la cuota del producto $i$ en las exportaciones al mercado $m$ del país $j$ y del total de países de la UE25 respecto del total de productos exportados por el país $j$ al mercado $m$,

$$
S_{j m i}=\frac{p_{j m i} x_{j m i}}{\sum_{i \in I_{i m}} p_{j m i} x_{j m i}} \quad S_{j m i}=\frac{p_{j m i} x_{j m i}}{\sum_{i \in I_{j m}} p_{j m i} x_{j m i}}
$$

Finalmente para cada uno de los países considerados se obtienen una serie de indicadores agregados calculando las medias geométricas de las descomposiciones obtenidas para cada uno de los mercados de destino. Así,

$$
\begin{array}{rlrl}
M I_{j} & =\prod_{m \in M_{-j}}\left(M I_{j m}\right)^{a_{j m}} & M E_{j} & =\prod_{m \in M_{-j}}\left(M E_{j m}\right)^{a_{j m}} \\
P_{j} & =\prod_{m \in M_{-j}}\left(P_{j m}\right)^{a_{j m}} & X_{j} & =\prod_{m \in M_{-j}}\left(X_{j m}\right)^{a_{j m}}
\end{array}
$$

La expresión $M_{-j}$ corresponde al conjunto de los 67 mercados destino de las exportaciones de los países de los cuales se utilizan datos, lógicamente con la excepción del propio país $j$ que se está considerando. El factor de ponderación $a_{j m}$ se define como la media logarítmica de $s_{j m}$ y $s_{k m}$

$$
a_{j m}=\frac{\frac{s_{j m}-s_{k m}}{\ln s_{j m}-\ln s_{k m}}}{\sum_{m \in M_{-j}} \frac{s_{j m}-s_{k m}}{\ln s_{i m}-\ln s_{k m}}}
$$

donde $s_{j m}$ y $s_{k m}$ son, respectivamente, la cuota que representa el mercado $m$ en el total de las exportaciones totales del país $j$ y del total de países de la UE25:

$$
S_{j m}=\frac{\sum_{i} p_{j m i} x_{j m i}}{\sum_{m} \sum_{i} p_{j m i} x_{j m i}} \quad S_{k m}=\frac{\sum_{i} p_{k m i} x_{k m i}}{\sum_{m} \sum_{i} p_{k m i} x_{k m i}}
$$

Los datos utilizados en esta investigación provienen de dos fuentes distintas. Por una parte, los datos correspondientes a las comunidades autónomas españolas provienen de las Estadísticas de Comercio Exterior de España que publica la Agencia Tributaria del Ministerio de Economía y Hacienda del Gobierno español. Por otra parte, los datos correspondientes a los países de la UE25 están incluidos en la base de datos «Intra- and extra-EU trade» elaborada por Eurostat, la oficina estadística de la Unión Europea. En ambos casos la información que se ha utilizado corresponde al año 2005 y está desagregada por productos siguiendo la clasificación arancelaria integrada de los países comunitarios (TARIC). Dada la naturaleza del trabajo pro- 
puesto en la presente investigación, sólo tiene sentido trabajar con la máxima desagregación de los productos de la Nomenclatura Combinada (8 dígitos), disponible en ambas bases de datos, que agrupa un máximo de 10.096 productos. Los datos se refieren tanto a valores en unidades monetarias como a cantidades en unidades físicas, lo que permite calcular los precios como valores unitarios de los bienes exportados. Tal como se ha mencionado anteriormente, los datos desagregados para las regiones españolas se refieren solamente a las seis comunidades más exportadoras, que suponen en conjunto más del $76 \%$ del total español. Las regiones no consideradas tienen un peso pequeño en el total de las exportaciones españolas (menos del $6 \%$ que corresponde a Castilla-León, la más importante).

La Tabla A.1 en el Anexo presenta para cada territorio exportador los índices correspondientes a la cuota exportadora (Cuota), los márgenes extensivo $(M E)$ e intensivo $(M I)$ y la descomposición en precios $(P)$ y cantidades $(X)$ de este último, tal como han sido definidos en esta sección. Asimismo la tabla incluye otras variables relevantes para el análisis posterior como son el PIB (medido de acuerdo a la paridad del poder adquisitivo) $(Y)$, número de trabajadores $(L)$ y el PIB por trabajador $(Y / L)$ de los diferentes países de la muestra. Estas tres variables se presentan en valores porcentuales sobre el total de los países de la UE25. Todos los datos vienen referidos al año 2005.

\section{Análisis de los resultados}

El contenido de los datos de la Tabla A.1 puede resumirse de forma clara con la ayuda de una serie de gráficos. En primer lugar, la Figura 1 presenta la relación entre la cuota exportadora y el tamaño de la economía para los 31 países considerados. El examen del gráfico parece confirmar que existe una correlación positiva entre exportaciones y PIB. De la misma manera, las Figuras 2 y 3 confirman que los dos márgenes exportadores definidos anteriormente, el extensivo y el intensivo, sustentan esta relación. 


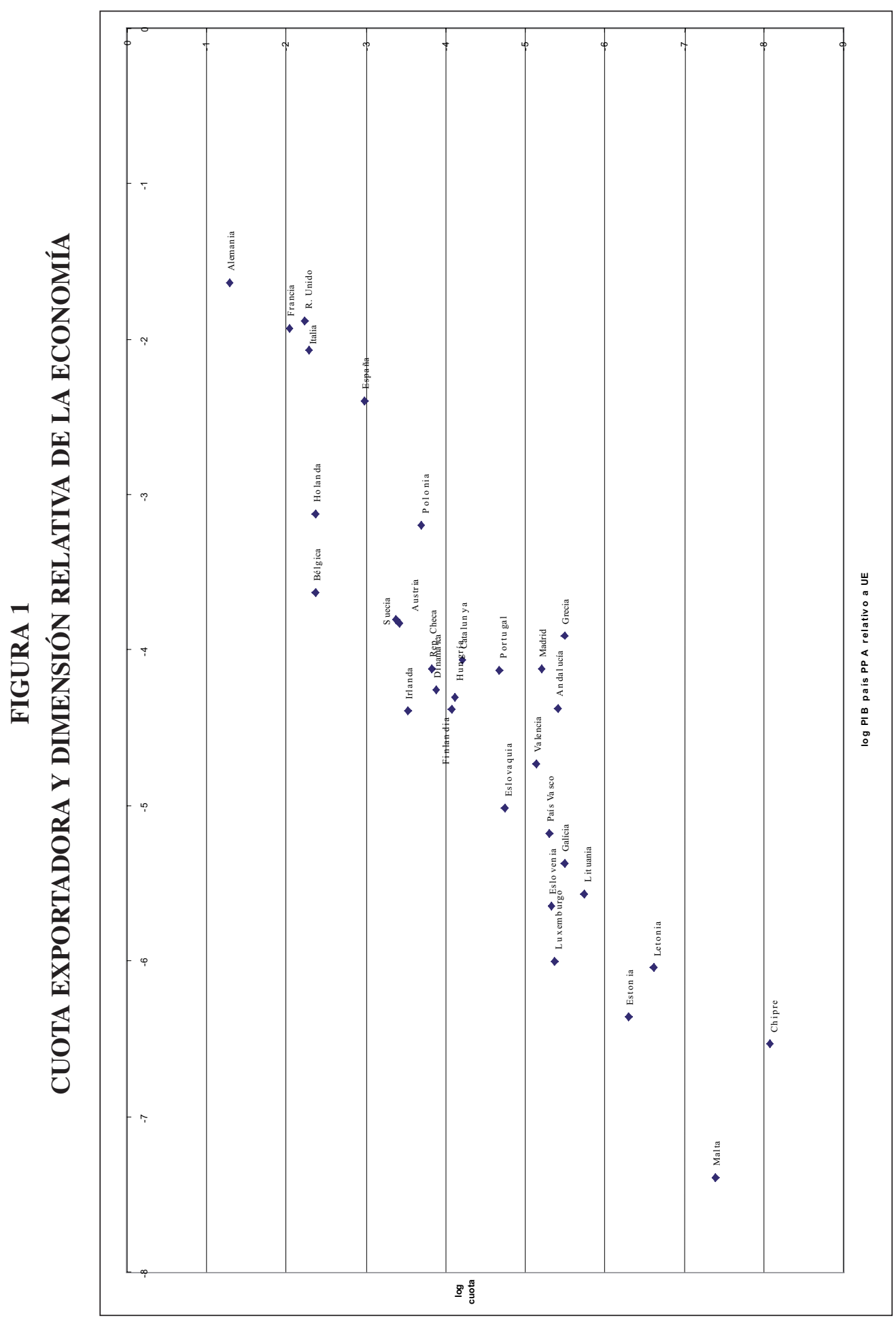




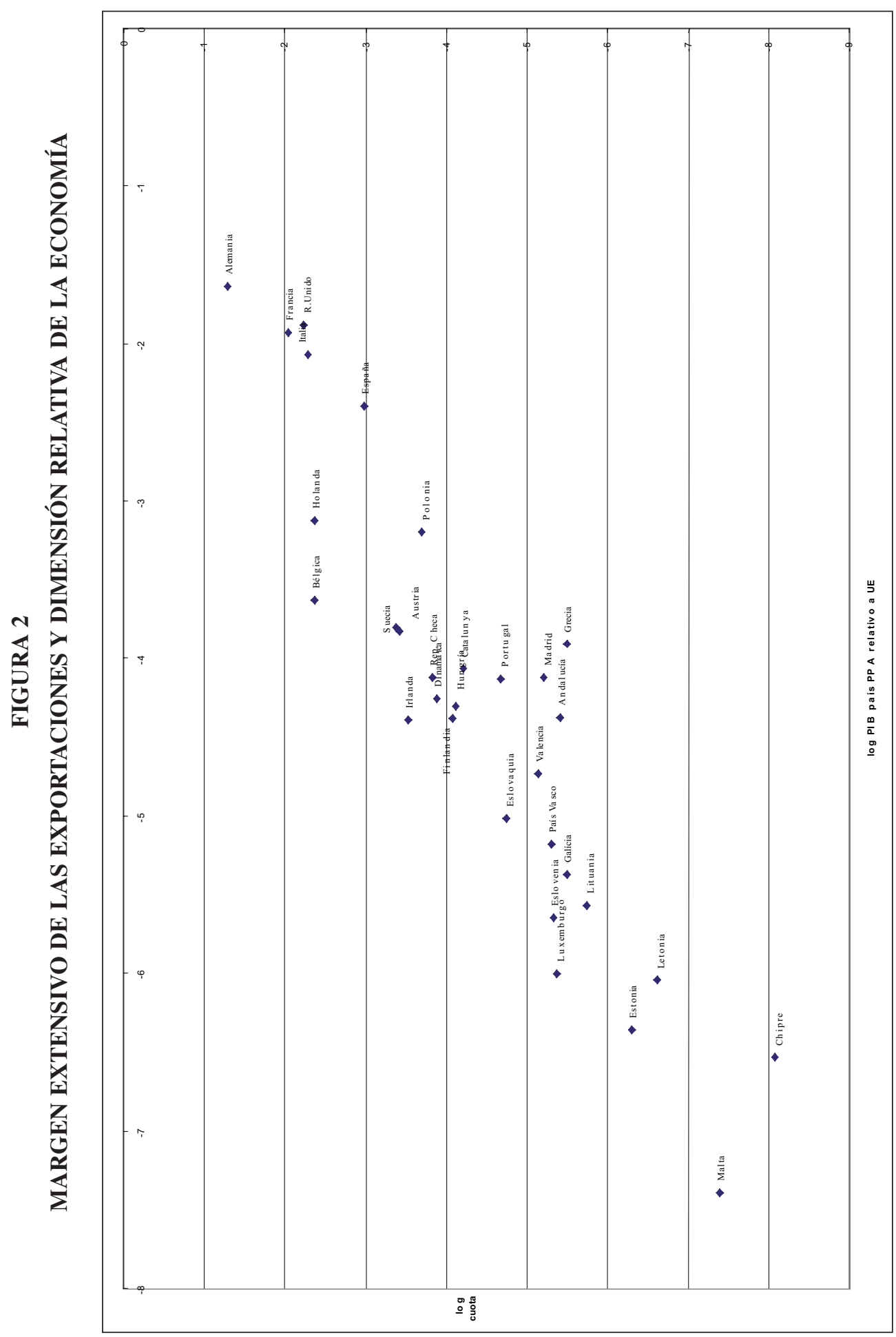




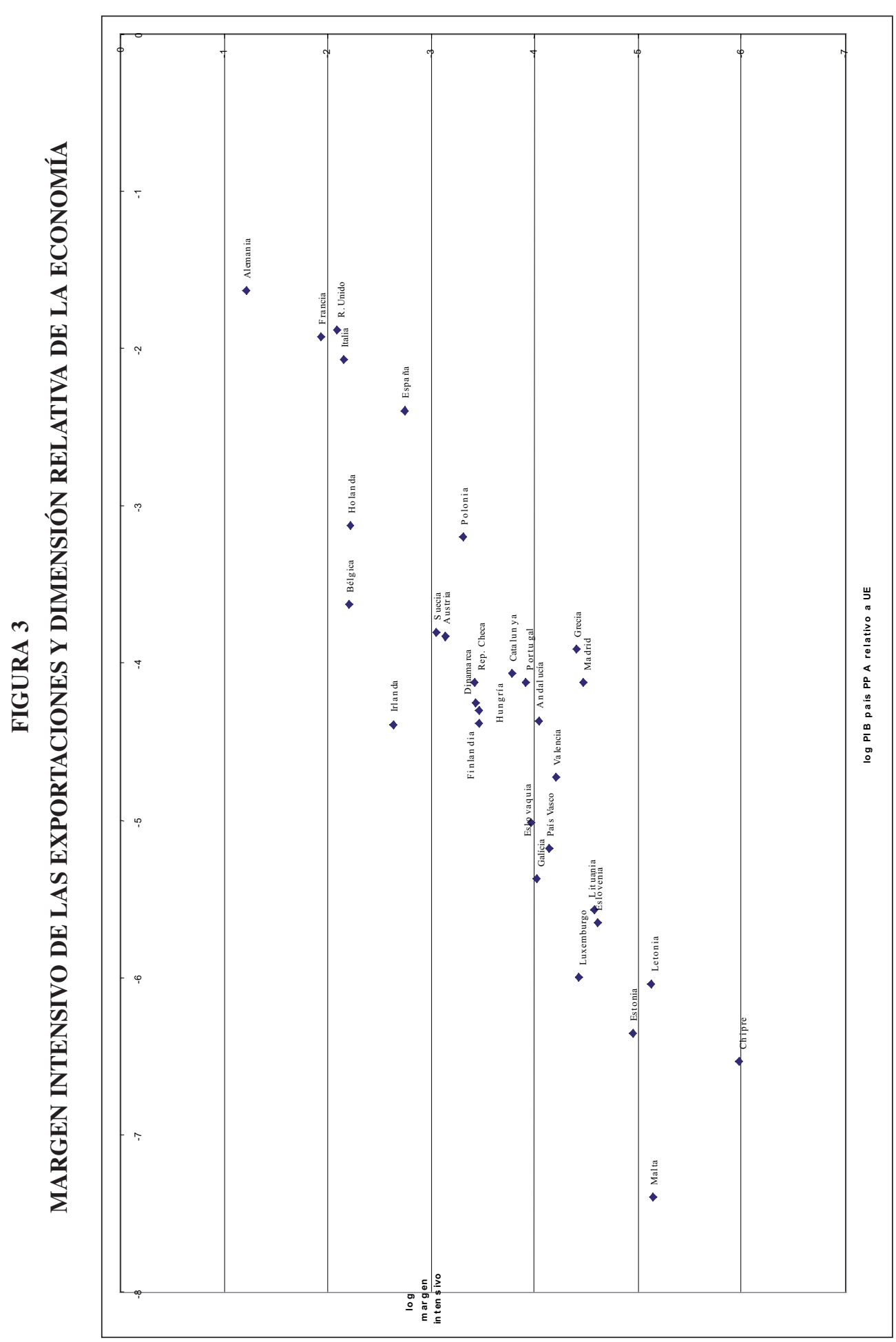


Para confirmar las intuiciones que se deducen de los gráficos y precisar la importancia que cada margen tiene en las exportaciones de las economías se procede al análisis de los resultados de la Tabla A.1 siguiendo la metodología apuntada en Hummels y Klenow (2005). En primer lugar, procedemos a la formalización de varios modelos de regresión en los que las variables a explicar son la cuota exportadora total de cada territorio y los márgenes que la componen y las variables explicativas son el PIB y sus componentes, número de trabajadores y PIB por trabajador. Todos los valores de las distintas variables están en logaritmos y las variables explicativas se expresan en términos relativos respecto del total calculado para todos los países europeos. El hecho de que MCO sea un operador lineal hace que los resultados de las regresiones presenten de forma aditiva la descomposición de los márgenes a través de los que las mayores economías exportan mayores cantidades. Cada regresión tiene 31 observaciones (los 25 países europeos y las seis regiones españolas $)^{8}$. Los resultados de este ejercicio vienen resumidos en la Tabla 1.

TABLA 1

FACTORES EXPLICATIVOS DE LA CUOTA EXPORTADORA Y SUS MÁRGENES

\begin{tabular}{|c|c|c|c|c|c|c|}
\hline \multirow[b]{2}{*}{ Variable dependiente } & \multicolumn{6}{|c|}{ Variable independiente } \\
\hline & $Y / L$ & & $Y$ & & $L$ & \\
\hline $\begin{array}{l}\text { Cuota exportadora } \\
\text { R-cuadrado ajustada }\end{array}$ & $\begin{array}{c}1,81 \\
(0,82) \\
0,11\end{array}$ & & $\begin{array}{c}1,03 \\
(0,08) \\
0,83\end{array}$ & & $\begin{array}{c}1,01 \\
(0,11) \\
0,73\end{array}$ & \\
\hline $\begin{array}{l}\text { Margen intensivo } \\
\text { R-cuadrado ajustada }\end{array}$ & $\begin{array}{c}1,27 \\
(0,56) \\
0,12\end{array}$ & $70,3 \%$ & $\begin{array}{c}0,69 \\
(0,06) \\
0,80\end{array}$ & $66,6 \%$ & $\begin{array}{c}0,67 \\
(0,08) \\
0,69\end{array}$ & $66,1 \%$ \\
\hline $\begin{array}{l}\text { Margen extensivo } \\
\text { R-cuadrado ajustada }\end{array}$ & $\begin{array}{c}0,54 \\
(0,30) \\
0,07\end{array}$ & $29,7 \%$ & $\begin{array}{c}0,35 \\
(0,04) \\
0,74\end{array}$ & $33,4 \%$ & $\begin{array}{c}0,34 \\
(0,04) \\
0,67\end{array}$ & $33,9 \%$ \\
\hline
\end{tabular}

Errores estándar entre paréntesis.

${ }^{8}$ Los resultados de las Tablas 1 y 2 no sufren cambios significativos cuando se excluye del análisis el dato referente a España, manteniendo los datos correspondientes a las regiones españolas. 
Los resultados obtenidos son en gran parte coincidentes con los procedentes del estudio de Hummels y Klenow (2005), aunque también deben resaltarse algunas importantes diferencias. La columna central de la Tabla 1 confirma que las mayores economías exportan sustancialmente mayores cantidades. Este resultado es, en términos cualitativos, idéntico al que se obtenía en Hummels y Klenow (2005), aunque las proporciones correspondientes a los márgenes intensivo y extensivo (dos terceras y una tercera parte en nuestro trabajo) eran muy distintas en el mencionado estudio, donde el margen extensivo era responsable del $62 \%$ de las mayores exportaciones de las economías más grandes. Por su parte, la primera y tercera columnas de la Tabla 1 vienen a confirmar que las economías con mayor número de trabajadores y con mayor producción por trabajador también tienen una mayor potencia exportadora. Las contribuciones de los márgenes extensivo e intensivo son parecidas a los correspondientes a la segunda columna, aunque es preciso especificar que el coeficiente correspondiente al margen extensivo en la primera columna (variable explicativa PIB por trabajador) es significativamente distinto de cero sólo a un nivel del $10 \%$.

En resumen puede afirmarse que los resultados de nuestro ejercicio difieren de los resultados del trabajo original de Hummels y Klenow (2005) no tanto en las previsiones acerca del mayor potencial exportador de las economías más grandes o más productivas, como en cuál es la contribución de los márgenes intensivo y extensivo a esas mayores exportaciones. El margen extensivo era, sin duda, el más significativo en el artículo de Hummels y Klenow (2005), mientras que en nuestro estudio, realizado a partir de una muestra de países notablemente diferente, el margen intensivo es el más importante. A la hora de analizar estas diferencias, hay que tener en cuenta la muy distinta composición de las muestras correspondientes a los dos estudios. La muestra considerada en este estudio está compuesta por un conjunto de países con un grado de desarrollo relativamente similar que, además, forman parte de un espacio económico común en el que comparten un gran número de características institucionales. La muestra de Hummels y Klenow (2005), con datos de 1995, incluye 126 países y es mucho más heterogénea en todos los sentidos.

La Figura 4 muestra la relación entre el margen cualitativo (variable $P$ en la Tabla A.1) y la dimensión relativa del PIB por ocupado ( $Y / L$ en la Tabla A.1) para las diferentes economías estudiadas. El gráfico parece confirmar el resultado obtenido por Hummels y Klenow (2005) de que los países más ricos (es decir, PIB por empleado más alto) tienden a exportar productos de mayor calidad y precio. 


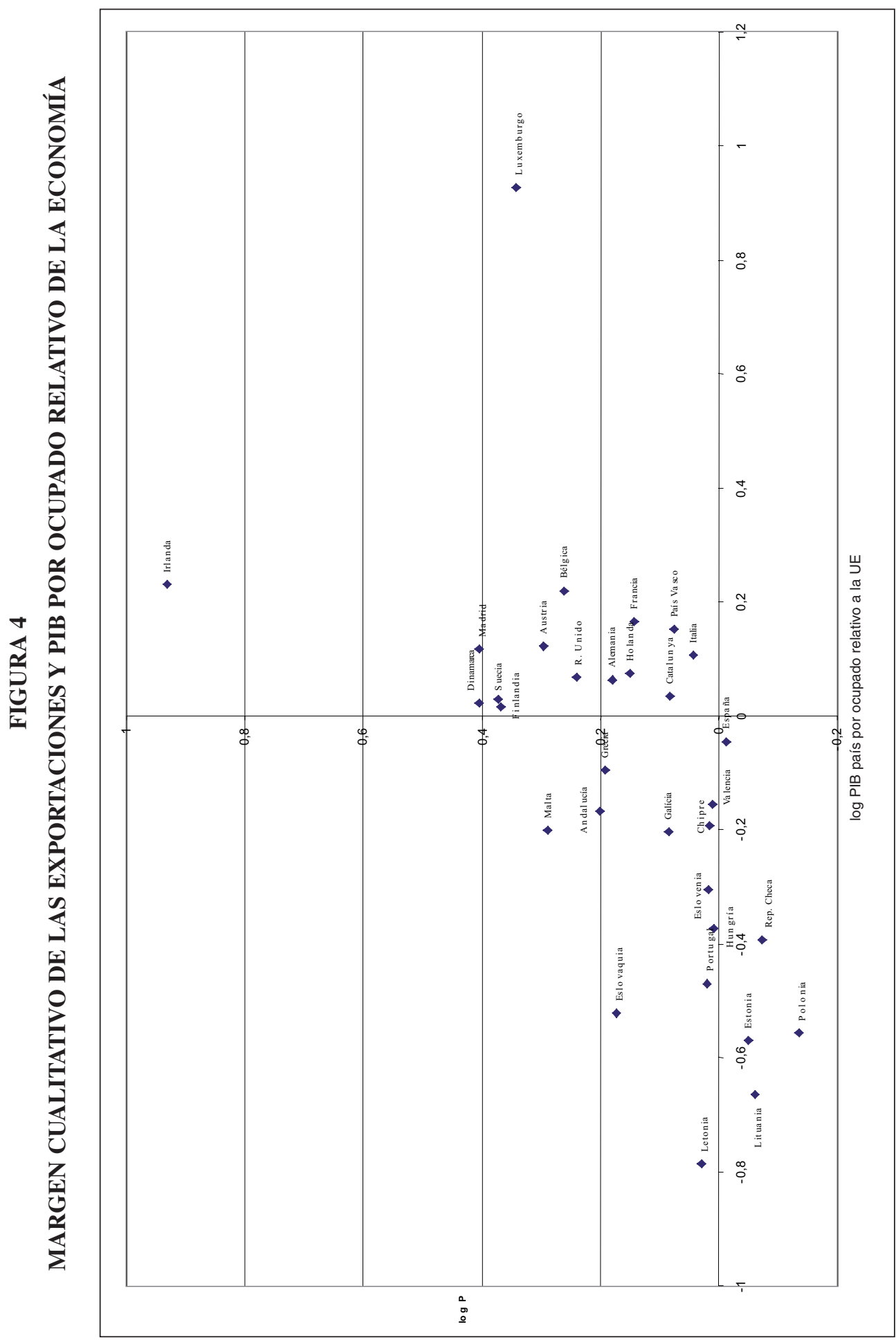


Esto último se puede afirmar de manera más precisa mediante la presentación de la descomposición del margen intensivo de las exportaciones en dos partes, precios y cantidades (Tabla 2). Lo que los resultados de esta tabla ponen de manifiesto es que las mayores economías, tanto en términos de PIB como de número de trabajadores, exportan mayores cantidades, pero a precios que no son significativamente distintos, que las economías más pequeñas. En cambio, las economías más ricas, en términos de mayor PIB por trabajador exportan cantidades que no son significativamente mayores (el coeficiente estimado no es significativamente distinto de cero), pero a precios más altos. En términos cuantitativos el resultado se puede resumir diciendo que economías más ricas, con un PIB por trabajador un $100 \%$ más elevado, exportan a precios que son un 36\% más altos. Este resultado no es de ningún modo sorprendente y es perfectamente compatible con la hipótesis avanzada en Schott (2004) de que los países más avanzados compiten con los países menos ricos en algunos segmentos de producción muy similares, aunque muchas veces con mayores niveles de calidad (y, por tanto precios más altos). Los resultados presentados en la Tabla 2 también son, en términos cualitativos, muy similares a los obtenidos en el trabajo de Hummels y Klenow (2005), con la salvedad de que en este trabajo también resultaban significativamente distintas de cero las mayores cantidades exportadas por los países de mayor PIB por trabajador.

TABLA 2

DESCOMPOSICIÓN DEL MARGEN INTENSIVO

\begin{tabular}{|l|ccc|}
\hline & \multicolumn{3}{|c|}{ Variable independiente } \\
\hline \multicolumn{1}{|c|}{ Variable dependiente } & $\boldsymbol{Y} / \boldsymbol{L}$ & $\boldsymbol{L}$ \\
\hline Precios & 0,36 & 0,01 & $-0,02$ \\
& $(0,09)$ & $(0,03)$ & $(0,03)$ \\
R-cuadrado ajustada & 0,31 & $-0,03$ & $-0,02$ \\
\hline Cantidades & 0,92 & 0,68 & 0,69 \\
R-cuadrado ajustada & $(0,56)$ & $(0,06)$ & $(0,07)$ \\
& 0,05 & 0,82 & 0,76 \\
\hline
\end{tabular}

Errores estándar entre paréntesis.

Por último, la Tabla 3 nos permite analizar la posición relativa de las economías regionales españolas. Para empezar, el panel A de la Tabla 3 nos muestra el valor esperado de la variable Cuota (cuota exportadora) cuando la variable explicativa es $Y$ y las discrepancias, en términos absolutos y relativos, con el valor observado correspondiente a España y las seis economías regionales españolas. Claramente se deriva de la observación del Gráfico 1 y de los datos expuestos en la Tabla 3 que la cuota exportadora correspondiente a España es mucho menor de lo que se deduciría atendiendo al tamaño relativo de su economía. Esta circunstancia se debe al comparativamente escaso peso exportador de todas las economías regionales, aunque el valor de las discrepancias para las observaciones de Madrid, Andalucía y Valencia es notablemente superior a las de Galicia, País Vasco y Cataluña. 
TABLA 3

VALORES AJUSTADOS Y DISCREPANCIAS PARA LOS DISTINTOS MÁRGENES

\begin{tabular}{|l|c|r|r|r|r|r|r|r|r|r|r|r|}
\hline & \multicolumn{3}{|c|}{ A } & \multicolumn{3}{c|}{ B } & \multicolumn{3}{c|}{ C } & \multicolumn{3}{c|}{ D } \\
\hline & $\begin{array}{c}\text { Ajuste } \\
\text { cuota }\end{array}$ & $\begin{array}{c}\text { Desvia- } \\
\text { ción }\end{array}$ & $\begin{array}{c}\text { \% } \\
\text { desv. }\end{array}$ & $\begin{array}{c}\text { Ajuste } \\
\boldsymbol{M I}\end{array}$ & $\begin{array}{c}\text { Desvia- } \\
\text { ción }\end{array}$ & $\begin{array}{c}\text { \% } \\
\text { desv. }\end{array}$ & $\begin{array}{c}\text { Ajuste } \\
\boldsymbol{M E}\end{array}$ & $\begin{array}{c}\text { Desvia- } \\
\text { ción }\end{array}$ & $\begin{array}{c}\text { \% } \\
\text { desv. }\end{array}$ & $\begin{array}{c}\text { Ajuste } \\
\boldsymbol{P}\end{array}$ & $\begin{array}{c}\text { Desvia- } \\
\text { ción }\end{array}$ & \multicolumn{1}{c}{$\begin{array}{c}\text { \% } \\
\text { desv. }\end{array}$} \\
\hline Andal. & 0,011 & $-0,007$ & $-61,7$ & 0,025 & $-0,008$ & $-31,6$ & 0,453 & $-0,200$ & $-44,1$ & 1,149 & 0,072 & 6,2 \\
Catal. & 0,016 & $-0,001$ & $-6,6$ & 0,031 & $-0,009$ & $-27,3$ & 0,504 & 0,143 & 28,5 & 1,234 & $-0,149$ & $-12,1$ \\
España & 0,089 & $-0,039$ & $-43,5$ & 0,099 & $-0,035$ & $-35,2$ & 0,899 & $-0,115$ & $-12,8$ & 1,200 & $-0,213$ & $-17,8$ \\
Galicia & 0,004 & 0,000 & $-0,5$ & 0,013 & 0,005 & 40,0 & 0,321 & $-0,093$ & $-28,9$ & 1,134 & $-0,047$ & $-4,1$ \\
Madrid & 0,015 & $-0,009$ & $-63,4$ & 0,030 & $-0,019$ & $-62,2$ & 0,494 & $-0,016$ & $-3,2$ & 1,271 & 0,225 & 17,7 \\
P. Vasco & 0,005 & 0,000 & $-0,5$ & 0,014 & 0,001 & 8,6 & 0,342 & $-0,029$ & $-8,3$ & 1,287 & $-0,209$ & $-16,3$ \\
Valenc. & 0,008 & $-0,002$ & $-27,3$ & 0,020 & $-0,005$ & $-25,2$ & 0,401 & $-0,011$ & $-2,8$ & 1,153 & $-0,145$ & $-12,6$ \\
\hline
\end{tabular}

La siguiente pregunta que pretende responder nuestro trabajo es cuál es el margen (intensivo o extensivo) responsable de estos resultados. Para ello, los paneles B y $\mathrm{C}$ de la Tabla 3 presentan la diferencia entre los valores esperados y observados para las estas variables $(M I$ y $M E$ ) cuando la variable explicativa es $Y$. Es sencillo llegar a la conclusión de que la economía española debe su baja cuota exportadora a que los dos márgenes, intensivo y extensivo, son bajos en términos comparativos. En términos generales, aunque con algunas variaciones, esto es también lo que corresponde a las regiones exportadoras españolas. Con mayor o menor intensidad, Andalucía, Madrid y Valencia presentan márgenes intensivos y extensivos por debajo de los esperados. La excepción a la regla general sería el margen extensivo para el caso de la economía catalana y el margen intensivo de las economías gallega y vasca, para las que se observan niveles por encima de los esperados en razón de la dimensión de su PIB.

Por último, el panel D de la Tabla 3 presenta las diferencias entre los valores esperados y observados para la variable $P$ cuando la variable independiente es $Y / L$ para las observaciones correspondientes a España y las economías regionales españolas. Incluso teniendo en cuenta que, tal como se muestra en la Tabla A.1, España presenta unos niveles de PIB por trabajador muy bajos en términos comparativos, de la Tabla 3 se deduce que el margen cualitativo de la economía española está por debajo de su valor esperado ${ }^{9}$. Debe destacarse, sin embargo, que este dato oculta cierto nivel de variabilidad entre las diferentes regiones españolas. Destacan los elevados valores observados del margen cualitativo correspondientes a la economía madrileña y, en menor medida, la economía andaluza. Al mismo tiempo, Cataluña, Galicia, el País Vasco y Valencia presentan valores del margen cualitativo que están claramente por debajo de lo cabría esperar atendiendo a los valores del PIB por trabajador de dichas comunidades.

${ }^{9}$ Esta observación es coincidente con la especialización en bienes de baja calidad de la economía española que se que se deduce del estudio de FONTAGNÉ, GAULIER y ZIGNAGO (2008) (véanse las Tablas 6 y 7 del trabajo). 
Para finalizar el análisis cabe resaltar dos consideraciones. En primer lugar, es destacable que todos los resultados referidos a la economía española presentados en este trabajo no difieren de forma muy radical de los obtenidos en Hummels y Klenow (2005), a pesar de los diez años transcurridos y de la importante tasa de crecimiento de las exportaciones españolas registradas durante este período ${ }^{10}$. En segundo lugar, nuestro trabajo no nos dice nada acerca de las posibles explicaciones de los resultados relativos a las economías regionales. Los estudios a nivel regional que aborden este problema son muy escasos (en este sentido cabe destacar los trabajos relativos a la calidad de las exportaciones de Milgram Baleix, 2002 para Andalucía y Fernández, 2006 para Cataluña) y creemos que este hecho justifica la necesidad de un mayor esfuerzo investigador en este área.

\section{Conclusiones}

A pesar del importante crecimiento de las exportaciones españolas experimentado en los últimos años, la economía española continúa registrando valores muy por debajo de la media de los países de la UE25 en el indicador Exportaciones / PIB. El presente trabajo nos permite analizar la contribución a este fenómeno de los diferentes márgenes (extensivo, intensivo y cualitativo) de las exportaciones españolas. A modo de resumen, puede afirmarse, en primer lugar, que la economía española exporta una menor variedad de productos (margen extensivo) que otros países europeos comparables. En segundo lugar, se demuestra que el valor de los productos efectivamente exportados es menor (margen intensivo). Finalmente, también se comprueba que el precio (valor unitario) de los productos exportados es menor comparado con los precios de exportación de países europeos similares (margen cualitativo). La utilización de los datos de las exportaciones españolas por regiones nos permite analizar este tipo de resultados desde una perspectiva más desagregada territorialmente. El resultado de nuestro análisis se puede resumir diciendo que, en general, no se aprecian grandes variaciones por territorios y que los márgenes exportadores de las regiones españolas son también comparativamente bajos, aunque cabe reseñar las excepciones correspondientes a los márgenes extensivo en Cataluña, intensivo en Galicia y el País Vasco y cualitativo en Madrid y Andalucía.

Todos estos resultados tienen un indudable interés para la formulación de la política económica y justificarían, desde nuestro punto de vista, la realización de nuevas investigaciones más detalladas que permitan conocer la evolución de los márgenes

${ }^{10}$ Con el objetivo de comprobar si la estructura sectorial de las economías y, en especial, el peso de los sectores de servicios y la construcción influye de manera determinante en los resultados presentados en el trabajo se estimaron las mismas regresiones utilizando como variables explicativas el valor añadido de los sectores primario y secundario en lugar del PIB total del país, el empleo de los mismos sectores en lugar del empleo total y el cociente entre ambas variables. Los resultados obtenidos no difieren de forma significativa de los presentados en este trabajo. 
exportadores a nivel de sectores y productos. Es indudable que, en un contexto internacional caracterizado por el protagonismo creciente de los intercambios intraindustriales, los márgenes extensivo (mayor o menor diversificación de las exportaciones) y cualitativo (especialización en variedades de mayor o menor calidad) son cada vez más importantes para el crecimiento de las exportaciones de los países más competitivos (tal como se demuestra en los trabajos de Schott, 2004 y Fontagné, Gaulier y Zignago, 2008).

\section{Referencias bibliográficas}

[1] AMURGO PACHECO, A. (2006).«Preferential Trade Liberalization and the Range of Exported Goods: The Case of the Euro-Mediterranean FTA», HEI Working Paper, 18/2006, Graduate Institute of International Studies.

[2] AMURGO-PACHECO, A. y PIÉROLA, M.D. (2007). «Patterns of export diversification in developing countries: intensive and extensive margins», HEI Working Paper, 20/2007, Graduate Institute of International Studies.

[3] ARMINGTON, P.S. (1969). «A Theory of Demand for Products Distinguished by Place of Production», IMF Staff Papers, 16 (1), p. 159-178.

[4] BALDWIN, R.E. y DI NINO, V. (2006). «Euros and zeros: The common currency effect on trade on new goods», NBER Working Paper, 12673.

[5] BESEDES, T. y PRUSA, T.J. (2006). «The Role of Extensive and Intensive Margins and Export Growth», Mimeo, Rutgers University.

[6] CHANEY, T. (2006). «Distorted Gravity: Heterogeneous Firms, Market Structure and the Geography of International Trade», Mimeo, University of Chicago.

[7] DEBAERE, P. y MOSTASHARI, S. (2005). «Do Tariffs Matter for the Extensive Margin of International Trade: An Empirical Analysis», CEPR Discussion Paper, 5620.

[8] EVENETT, S.J. y VENABLES, A.J. (2002). «Export Growth in Developing Countries: Market Entries and Bilateral Trade Flows», Mimeo. Disponible en www.evenett.com

[9] FABRIZIO, S., IGAN, D. y MODY, A. (2007). «The Dynamics of Product Quality and International Competitiveness», IMF Working Paper WP/07/97.

[10] FARUQ, H. (2006). «New Evidence on Product Quality and Trade», Center for Applied Economics and Policy Research CAEPR Working Paper 2006-019, Indiana University.

[11] FEENSTRA, R.C. y KEE, H.L. (2007). «Trade Liberalisation and Export Variety: A Comparison of Mexico and China», The World Economy, 30 (1), p. 5-21.

[12] FELBERMAYR, G.J. y KOHLER, W. (2006). «Exploring the Intensive and Extensive Margins of World Trade», Review of World Economics, 142 (4), p. 642-674.

[13] FERNÁNDEZ, T. (2006). «La competitivitat de les manufactures catalanes a la Unió Europea ampliada des de la perspectiva del quality gap». Tesis doctoral en la Universitat Autònoma de Barcelona, noviembre de 2006.

[14] FLAM, H. y NORDSTRÖM, H. (2006). «Euro Effects on the Intensive and Extensive Margins of Trade», CESifo Working Paper, 1881.

[15] FONTAGNÉ, L., GAULIER, G. y ZIGNAGO, S. (2008). «Specialization across varieties and North-South competition», Economic Policy, 53, p. 51-91. 
[16] FUNKE, M. y RUHWEDEL, R. (2003). «Trade, product variety and welfare: A quantitative assessment for the transition economies in Central and Eastern Europe», Bank of Finland Institute for Economies in Transition, BOFIT Discussion Paper, 17/2003.

[17] GROSSMAN, G.M. y HELPMAN, E. (1991). Innovation and growth in the global economy, Cambridge. MIT Press.

[18] KANG, K. (2004). «The Path of the Extensive Margin (Export Variety): Theory and Evidence», Mimeo, University of California, Davis.

[19] HELPMAN, E., MELITZ, M. y RUBINSTEIN, Y. (2007). «Estimating Trade Flows: Trading Partners and Trading Volumes», NBER Working Paper, 12927.

[20] HILLBERRY, R.H. y C.A. MCDANIEL (2002). «A Decomposition of North American Trade Growth since NAFTA», U.S. International Trade Commission, Office of Economics Working Paper, 2002-12-A.

[21] HUMMELS, D. y KLENOW, P.J. (2002). «The Variety and Quality of a Nation's Trade», NBER Working Paper, 8712.

[22] HUMMELS, D. y KLENOW, P.J. (2005). «The Variety and Quality of a Nation's Exports», American Economic Review, 95 (3), p. 704-723.

[23] KEHOE, T.J. y RUHL, K.J. (2003). «How Important is the New Goods Margin in International Trade?», Federal Reserve Bank of Minneapolis, Research Department Staff Report, 324.

[24] KRUGMAN, P.R. (1981). «Intraindustry Specialization and the Gains from Trade" Journal of Political Economy, 89 (5), p. 959-973.

[25] MELITZ, M.J. (2003). «The Impact of Trade on Intra-industry Reallocations and Aggregate Industry Productivity», Econometrica, 71 (6), p. 1695-1725.

[26] MILGRAM BALEIX, J. (2002). «La importancia de la calidad en el comercio de Andalucía con la UE», Boletín Económico de Andalucía, 31-31, p. 247-260.

[27] SCHOTT, P.K. (2004). «Across-Product versus Within-Product Specialization in International Trade», Quarterly Journal of Economics, 119 (2), p. 647-678. 


\section{ANEXO}

TABLA A.1

PRINCIPALES RESULTADOS DE LA DESCOMPOSICIÓN DE LOS MÁRGENES EXPORTADORES

\begin{tabular}{|c|c|c|c|c|c|c|c|c|}
\hline País & Cuota & $M E$ & $M I$ & $\boldsymbol{P}$ & $X$ & $\boldsymbol{Y}$ & $L$ & $Y / L$ \\
\hline ALEMANIA & 0,273 & 0,927 & 0,295 & 1,196 & 0,246 & 0,196 & 0,184 & 1,066 \\
\hline ANDALUCIA & 0,004 & 0,253 & 0,017 & 1,221 & 0,014 & 0,013 & 0,015 & 0,848 \\
\hline AUSTRIA & 0,032 & 0,758 & 0,043 & 1,341 & 0,032 & 0,022 & 0,019 & 1,131 \\
\hline BELGICA & 0,093 & 0,853 & 0,109 & 1,297 & 0,084 & 0,027 & 0,021 & 1,248 \\
\hline CATALUÑA & 0,015 & 0,647 & 0,023 & 1,085 & 0,021 & 0,017 & 0,017 & 1,035 \\
\hline CHIPRE & 0,000 & 0,124 & 0,003 & 1,015 & 0,002 & 0,001 & 0,002 & 0,827 \\
\hline DINAMARCA & 0,020 & 0,635 & 0,032 & 1,496 & 0,021 & 0,014 & 0,014 & 1,025 \\
\hline ESLOVAQUIA & 0,009 & 0,459 & 0,019 & 1,188 & 0,016 & 0,007 & 0,011 & 0,594 \\
\hline ESLOVENIA & 0,005 & 0,485 & 0,010 & 1,015 & 0,010 & 0,004 & 0,005 & 0,737 \\
\hline ESPAÑA & 0,050 & 0,784 & 0,064 & 0,986 & 0,065 & 0,092 & 0,096 & 0,957 \\
\hline ESTONIA & 0,002 & 0,259 & 0,007 & 0,951 & 0,007 & 0,002 & 0,003 & 0,567 \\
\hline FINLANDIA & 0,017 & 0,547 & 0,031 & 1,447 & 0,021 & 0,013 & 0,012 & 1,032 \\
\hline FRANCIA & 0,130 & 0,904 & 0,143 & 1,153 & 0,124 & 0,147 & 0,124 & 1,181 \\
\hline GALICIA & 0,004 & 0.228 & 0,018 & 1,088 & 0,016 & 0,005 & 0,006 & 0,817 \\
\hline GRECIA & 0,004 & 0,334 & 0,012 & 1,208 & 0,010 & 0,020 & 0,022 & 0,911 \\
\hline HOLANDA & 0,093 & 0,860 & 0,108 & 1,159 & 0,093 & 0,044 & 0,041 & 1,079 \\
\hline HUNGRIA & 0,016 & 0,524 & 0,031 & 1,007 & 0,031 & 0,014 & 0,020 & 0,690 \\
\hline IRLANDA & 0,029 & 0,411 & 0,071 & 2,536 & 0,028 & 0,012 & 0,010 & 1,261 \\
\hline ITALIA & 0,101 & 0,874 & 0,115 & 1,042 & 0,110 & 0,127 & 0,114 & 1,113 \\
\hline LETONIA & 0,001 & 0,227 & 0,006 & 1,028 & 0,006 & 0,002 & 0,005 & 0,456 \\
\hline LITUANIA & 0,003 & 0,311 & 0,010 & 0,939 & 0,011 & 0,004 & 0,007 & 0,515 \\
\hline LUXEMBURGO & 0,005 & 0,386 & 0,012 & 1,405 & 0,008 & 0,002 & 0,001 & 2,534 \\
\hline MADRID & 0,005 & 0,478 & 0,011 & 1,497 & 0,008 & 0,016 & 0,014 & 1,126 \\
\hline MALTA & 0,001 & 0,104 & 0,006 & 1,333 & 0,004 & 0,001 & 0,001 & 0,819 \\
\hline PAIS VASCO & 0,005 & 0,314 & 0,016 & 1,077 & 0,015 & 0,006 & 0,005 & 1,164 \\
\hline POLONIA & 0,025 & 0,684 & 0,036 & 0,871 & 0,041 & 0,041 & 0,071 & 0,574 \\
\hline PORTUGAL & 0,009 & 0,464 & 0,020 & 1,018 & 0,019 & 0,016 & 0,026 & 0,626 \\
\hline REINO UNIDO & 0,106 & 0,867 & 0,122 & 1,270 & 0,096 & 0,153 & 0,143 & 1,072 \\
\hline REPÚBLICA CHECA & 0,021 & 0,665 & 0,032 & 0,929 & 0,035 & 0,016 & 0,024 & 0,676 \\
\hline SUECIA & 0,034 & 0,715 & 0,047 & 1,442 & 0,033 & 0,022 & 0,022 & 1,019 \\
\hline VALENCIA & 0,006 & 0,390 & 0,015 & 1,009 & 0,015 & 0,009 & 0,010 & 0,857 \\
\hline
\end{tabular}

Notas: Todas las variables corresponden al año 2005.

Cuota $=M E^{*} M I$

$M E=$ Margen extensivo

$M I=$ Margen intensivo

$P=$ Precios

$X=$ Cantidades

$Y=$ Porcentaje PIB-PPA del país relativo al total de la UE

$L=$ Porcentaje ocupación del país relativo al total de la UE

$Y / L=$ Cociente de los dos porcentajes anteriores

Fuentes: Eurostat, Contabilidad Regional INE y elaboración propia. 